\title{
Pulverizing the Monopoly of Mind: Three Roles of the Body in Cognition
}

\author{
Akhil Kumar Singh*
}

\begin{abstract}
Traditionally, cognition was understood as a computational process in the brain. Conventional views are inclined towards the existence of discrete and internal representations realised by highly specific mechanisms in the brain. The embodied approach challenges this view and accepts the evolution of cognitive abilities. There is a shift in focus from considering the brain as solely responsible for cognition to believing that the body is deeply integrated in cognitive functioning. However, it does not deny the central position of the brain in the process of cognition but considers the possibility of including other factors. At the basic level, there are three ways in which an agent's body can be utilised for the cognitive process. An agent's body may help to generate, operate and distribute the cognitive processes. This perspective diminishes the monopoly of the brain by taking into account the importance of the body and the environment for cognition.
\end{abstract}

Keywords: Embodiment, Cognition, Mind, Brain, Embodied

\section{Introduction}

Embodied cognition is a recent emerging viewpoint in cognitive science which holds that cognitive processes are deeply rooted in an agent's bodily interaction with the world (Wilson, 2002). It has

* IIT Jodhpur, India; singh.35@iitj.ac.in 
been argued that humans have evolved from organisms whose neural resources were linked mainly with the perceptual and sensory-motor processing and whose cognitive activities were related to the immediate interaction with the environment. For many decades, cognition has been viewed as a sort of computational process in the brain. The brain plays a central role but embodied cognition minimises the role of the brain in cognitive processing. Traditionally, the mind was viewed as an abstract information processor which had little connection with the outside world and perceptual and motor systems were not considered relevant to understanding central cognitive processes. They were kept in the periphery of the cognitive processes and taken into account only as input and output devices.

Conventional views are inclined towards the existence of discrete and internal representations realised by highly specific mechanisms in the brain. It was assumed that these mechanisms were shaped by natural evolution and coded in our genetics. This view leads to individualism or internalism which claims that cognition is supervened on the internal nature of the cogniser. This type of research methodology which is solely based on the internal nature is called 'Methodological Solipsism' (Fodor, 1980). In this way, all core topics of cognitive neuroscience were accounted locally without challenging the boundaries of the brain-skull. Even if there has been consideration of moving beyond this boundary, they have been limited in their consideration by including sensory input and behavioural output only. Traditional views of the mind and cognition were dedicated towards the 'classical sandwich model' (Hurley \& Noe, 2003), where mind/cognition is sandwiched between perception (input) and action (output). The embodied cognitive approach accepts cognition as a result of active and dynamic interaction between an agent's neural and non-neural processes. It does not make a watertight bifurcation between cognition, agent's bodily experience and real-life situations. Looking at how the body performs these functions in the external world provides evidence that cognition is neither bounded by the brain, nor by the body itself.

Earlier, cognitive processes (traditional approach towards cognition) were treated to be autonomous, occurring in the brain 
and alienated from the world. But, with the advancement in scientific experiments, it has been found that the brain is only one part of the whole cognitive system. It is more like the CPU of a computer, the main device for processing. However, the result of that processing is dependent on and influenced by inputs and outputs. Similarly, the brain also processes upon the inputs in the form of stimulus received from the world through sense organs and exerts the output in the form of motor actions. Initially, when a child is born, the pre-stored concepts in the brain are missing but it receives all this input in the form of stimulus which essentially organises the brain. In the initial phase, the regions of the brain are not actually pre-determined. Mriganka Sur's experiment also proved that "experience shapes the brain" not "by the brain" (Roe, Pallas, Kwon, \& Sur, 1992). In this experiment; they rewired the brain of a ferret so that the information from its retina was plugged into its auditory cortex. The assumption was that the animal would be blinded but ferrets could still see. Furthermore, their auditory cortex now resembled the typical ferret visual cortex, complete with spatial maps and neurons tuned to detect light. This shows that there is nothing special in the cells in the visual cortex that makes them visual. Therefore, it was concluded that the areas of the brain responsible for a particular cognitive process can be altered. This 'neural plasticity' shows that the association of a particular brain area with a cognitive activity is not fixed; they coevolve. The different brain areas are structured according to their ability to perform functions. For example, language skills do not pre-exist. Thus, the part of the brain responsible for it is not specifically created for it; it is just used for it. Hence, the idea that everything is pre-programmed, like the pre-made house blocks that we suddenly put together to construct houses in one day, is not how the brain works. The brain is like houses that we construct from scratch- step by step.

\section{What is Embodied Cognition?}

Embodied cognition is an idea that shifts focus from the thesis that the brain is the solely responsible resource available for cognition to the thought that the body is deeply integrated into the cognition. Embodied cognition does not deny the central position of the brain 
in the process of cognition but opens up the door to other factors for integration. In this sense, it is not like a well-defined theory but a research programme. The embodiment thesis is based on the assumption that the interaction of mind, body and the environment is essential to cognition. An agent's bodily interaction with the world enables it to conceive and perceive the world. We think because we have inputs from the outside world to ponder upon.

Wilson and Foglia (2011)states, "cognition is embodied when it is deeply dependent upon features of the physical body of an agent, that is, when aspects of the agent's body beyond the brain play a significant causal or physically constitutive role in cognitive processing"(Wilson \& Foglia, 2011). In this definition, two phrases are important because they form two major claims for the embodied cognition: 'agent's body beyond the brain' and 'causal or constitutive role'. Earlier, before the emergence of the embodied cognition as a concept, the body was not taken into account for the understanding of the nature of mind and cognition, and the view that the think tank was situated inside the skull was dominant. So, the idea of embodied cognition mainly attacks this solipsistic view of cognition (Fodor, 1980). Embodied cognition also argues about the body's causal role in shaping the mind. It simply means that mental states (particularly cognitive process) are not just the brain possesses but it is the result of an agent's body and its interaction with the environment.

\subsection{Cognition as an Adaptive Evolution}

Embodied cognition accepts cognition as an adaptive evolution process within the biological theory of evolution. It argues that cognition evolved in organisms to interact with the environment in a better manner. It provided the tools to cope up with the everchanging environment and for their survival. Michael Anderson says that "Embodied cognition treats cognition as a set of tools evolved by organisms for coping with their environment" (Anderson, 2005). Each key term (tools, evolve, organisms, cope, environment) in this defining line is important and plays a significant role in understanding embodied cognition as an adaptive phenomenon. Cognition as a skill has an evolutionary history. This implies that cognition is adapted by the evolving 
organisms. It also evolves as it helps organisms to enhance their survival by gaining better control over their dynamic environment. Most organisms are concerned with survival and reproduction. With the evolution of cognitive capacity, they can take advantage of the diverse features of the environment for their utility. Humans are at the peak of evolution and cognitive capacity and therefore they have much greater control over the environment to utilise and change it according to their need.

Cognition co-evolved in organisms with specific emotional and physical characteristics. Emotions worked as an important selfregulatory aspect of embodied cognition. Emotions in a way worked as a motivator towards goal-relevant actions which helped in driving adaptive behaviour. This resulted in greater interaction and influence over the world and other organisms. Certain bodily features of organisms take advantage of cognitive processes. Every organism extensively uses their body structures to enhance the survival and activity in the environment. Our specific anatomy leads to the variety of things we can do. In particular, humans are fairly distinct in their ability to create tools and languages. The concept related to this adaptive cognition and anatomy is 'Affordance'.

Affordance is just the usage of an object based on our ability to interact with it. For instance, a teacup has the affordance of being grabbed because of its handle. It fits into the hand (palm) and one can drink from it because it has a rim. So before designing an object, one has to keep in mind the ways in which it would be used by humans based on their bodily abilities. Humans have ten fingers, five on each hand, large palms, arms that have a certain degree of movement and so on. All these bodily features help us to interact with the environment and take part in the cognitive processes. Thus it can be said that cognition is a mix of cognitive tools, (specific interaction with the world and co-operative interaction with the other organisms) that evolved due to the adaptive nature of the organism for enhancing their status of survival and controlling the surroundings for their benefit.

Embodied cognition was a response to the idea that mental states are pre-made and fitted into the brain but mind (mental states) is not pre-made. In the words of Varela, the traditional approach 
treated cognition as the representation of a pre-given world by a pregiven mind, but the embodied approach considers it as the enactment of a world and a mind through various actions that are performed by a being-in-the-world (Varela, Thompson, \& Rosch, 1992). We are situated in the world. We use our bodies to interact with the world. Therefore, cognition is formed on the basis of both the body and the world. In this sense, our cognition behaves like a dynamical system.

\subsection{Embodied Cognition as a Dynamical System}

The main approach is that cognitive systems are dynamical agents (Smith, 2005). This provides a break from the Cartesian legacy which argues that the brain is solely responsible for all mental states which in turn can affect bodily states. On the other hand, the dynamical system provides a view that all that is associated with the term 'mind' is fully incorporated into the natural world. A dynamical system is one which performs its activities and produces the result without having the conception of any central controller. They perform their activity by coupling with the various parts of the system. They are so coupled that any minor changes in a part can affect the overall performance of the whole system. This dynamical system approach for psychological processes is inspired by the Watt's Governor Model which argues that the mind is not a single entity. It is coupled with the environment and hence behaves like a system.

Dynamicists try to minimise and even sometimes deny the need for a centralised representational processing unit. They counter the traditional representational idea which says that an internal model is capable of producing the external structures that can be used by the cognitive agent to guide the behaviour of the agent. But dynamical theory tries to explain the agent's behaviour in terms of embodiment and embeddedness. It argues that we can discard the notion of abstract symbolic description if an agent can sense its world and allows its body to be directly influenced by the world.

\section{Major Claims/Support for Embodied Cognition}

Embodied cognition is a relatively recent comprehensive framework for the study of the mind. This approach has recently 
attained high acceptance in the cognitive psychology arena. There is a growing idea that the mind must be understood in the context of an agent's relationship and interaction with the world. There has been a lot of support coming in from the experimental procedures in psychology where the psychologists ask subjects to perform specific tasks by manipulating their bodies in certain ways. They find that the subjects differ and the results depend on simple facts such as whether they are right or left handed. There is also work in robotics where robots have been designed to perform complex tasks like navigating through a room and so on in a way that does not rely on computationalism that once was thought to be the only way out.

There is an obvious line of thought that cognition happening in a particular situation must deal with the constraints of 'real-time' pressure. An organism in a real environment has to act fast to cope up with the potential hostile and changing environment, predators, stationary objects, and other threats. This claim also counters the traditional representational view. In the real world, situations demand fast and continuously evolving response. There is limited time available to an organism to build up a comprehensive mental model for motor action. But it is argued that being situated in a condition, the agent requires efficient ways of generating situation appropriate action on the go. All creatures have cognitive limitations. Humans have limited information-processing abilities like limits of attention, working memory and so forth. Therefore, to cope up with the fast-changing environment, help must be taken from other available resources. Humans must use the environment itself to reduce their cognitive workload. An organism can overcome their cognitive load in two ways: 'using features of the stable environment' and by 'changing environment itself' in their favour. For example, hunting animals can use scent trails or visual signs like burrow holes to find their desired prey (stable environmental feature), using fingers or pen-paper to ease the counting processes (changing the environment). In these ways, an organism can take advantage to enhance the ability to interact and change its environment. Cognitive processes involve very complex and dense information flow between the brain and the world. Many scientific and behavioural experiments show that these complex operations cannot be performed by the brain alone. It is 
done by the brain, body and environment altogether. Factors responsible for the cognitive activities do not reside within the boundary of the skull but they are distributed across the individual and the situation in which it interacts. It behaves as a single coupled system where the cogniser and the cognitive environment get integrated.

The main function of the mind is to guide actions through cognitive mechanisms and it should be understood in terms of contributing to an agent's behaviour. The cognitive system is evolved because it is a behavioural control system which is an important trait for adaptation. Cognitive systems should be studied in terms of their functions in serving. Therefore, it is hard to alienate the world from the cognitive system. For example, the traditional view of visual perception proposes that the visual system present for the building of internal representation of the perceived world is used by some higher mechanisms in the brain. However, it has now been understood that visual perception is used for guiding actions. For instance, there is a study by David Kirsh and Paul Maglio on the Tetris game (Kirsh \& Maglio, 1994). In the Tetris game, falling block shapes must be rotated and horizontally fitted as compact as possible along with the shapes already fallen. This decision of rotation should be made early before the block falls far down to allow possible movements. The data shows that players use actual rotation and translation movements to simplify the problem to be solved, rather than computing a solution in mind first and then executing it. This clearly shows that visual cognition is used in guiding action and not merely for creating representations in the brain (Wilson, 2002).

Margret Wilson's 'six views of embodied cognition' provides a very comprehensive account of the topic (Wilson, 2002). It covers most, if not all, possible aspects in which cognitive activity occurs. The crux of the whole discussion is that the characteristics of cognition are dependent upon the characteristics of the physical body of the agent. These beyond, the brain-body features play a causal and constitutive role in the cognitive processes. This is in sharp distinction with the view that mind is the dominant entity in cognitive processes. Now, there is a need to specify the nature of the dependence of cognition on the body. What role does the body 
play on an agent's cognition? How does a body prove useful in the process of cognition? At the most general level, it can be stated that there are three distinct roles which a body plays in the whole cognitive process. Sometimes, a body helps to generate some content for cognition. It helps to distribute some cognitive processes and operates the overall cognitive activity as well. Talking metaphorically, body behaves as GOD for cognition: 'G' as 'Generator', 'O' as 'Operator' and ' $\mathrm{D}$ ' as 'Distributor'.

\section{a. Body as a generator}

An agent's body is an important part of cognition but it sets a limit too. There is a strong relationship between the kind of body an organism possesses and the concepts an organism can acquire. A particular cognition can seem easier or complex depending upon the agent's bodily characteristics. For instance, there is a difference between how humans and bats perceive the world. This difference in cognition arises due to differences in bodily characteristics, particularly sense organs. Sometimes particular characteristics of an organism can give rise to very different cognitive content which humans can never experience. In humans, the gesture is one such bodily feature through which they create and understand several concepts thereby enhancing their overall cognitive ability.

\section{b. Body as an operator:}

An agent's body functions in many ways to control and operate cognitive activities. It regulates cognition activities over space and time. As already stated, one of the main purposes of cognition is to perform actions to cope with the environment. An agent's body functions to regulate the co-ordination between cognition and action. Bodily structures of the agent facilitate real-time execution of complex behavioural activities in response to complex and fastchanging environmental events. The body functions to extract information from the world in the form of cognition and later delivers the output into the world in the form of behaviour or action. The body plays a feedback-driven role in cognitive processes. In this case, the body is more like a dynamical system which performs their functions without any central controller, solely based on the external stimulus. 


\section{c. Body as a distributor:}

An agent's body serves to distribute cognitive tasks between the brain and the body. The body helps to distribute computational and representational load between the neural (brain) and the nonneural (body) structures. The traditional view of cognition (that cognition is confined to the brain areas only) can be challenged by this function. Body as a distributor implies that cognition is not bounded by the skull and is related tobeyond-the-body environment. The body helps to offload (distribute) the cognitive work and ease the whole cognitive process. Offloading would mean taking an internal mental task and giving it a physical form outside of the brain. It comes handy when we have a difficult task at hand. Offloading essentially extends the mental capacity thereby increasing retention. Offloading can be extended to gestures also. Offloading is important during note-taking when internal thoughts are transferred into the physical world via pen and paper.

\section{Conclusion}

Conventional cognitive science is based on two assumptions which include the understanding that there is an impoverishment of stimulus data and there is a need for an internal representational calculation on stimulus data. Embodied cognition overcomes these assumptions and counts all the resources available in the environment that might support complex behaviour in real-time. The important result of this line of research is that we are able to use and produce all kinds of complex behaviour. This understanding removes the necessity of any central representational entity to explain cognition and behaviour. Our cognition is the result of bridging of three basic units: brain, body, and the environment. The body can participate in cognitive processes in the most engaging way i.e. to help in generating content for cognition, to distribute cognitive tasks over the body and beyond the body, and to coordinate cognitive processes itself. These are some ways by which mental tasks are taken and put into the physical world in order to ease survival and ensure better coping with the surrounding. 


\section{Acknowledgements}

With a great sense of joy and pleasure, I take this opportunity to thank my research supervisor, Dr Hari Narayanan V (IIT Jodhpur, India), for his valuable comments and suggestions on the initial version of this paper. My sincere thanks also goes to the anonymous referees of the Tattva - Journal of Philosophy for their valuable corrections, remarks, and suggestions.

\section{References}

Anderson, M. L. (2005). How to study the mind: An introduction to embodied cognition.In Embodied cognition and perceptual learning in adaptive development. Cambridge Scholars Press.

Fodor, J. (1980). Methodological Solipsism Considered as a Research Strategy in Cognitive Science. Behavioral and Brain Sciences, 3(1), 63-73.

Hurley, S., \& Noe, A. (2003). Neural Plasticity and Consciousness. Biology and Philosophy, 18(1), 131-168.

Kirsh, D., \& Maglio, P. (1994). On Distinguishing Epistemic from Pragmatic Action. Cognitive Science, 18(4), 513-549.

Lakoff, G., \& Johnson, M. (1999). Philosophy in the Flesh: the Embodied Mind $\mathcal{E}$ its Challenge to Western Thought. Basic Books.

Roe, A. W., Pallas, S. L., Kwon, Y. H., \& Sur, M. (1992). Visual Projections Routed to the Auditory Pathway in Ferrets: Receptive Fields of Visual Neurons in Primary Auditory Cortex. The Journal of Neuroscience, 12(9), 3651-3664.

Shapiro, L. (2010). Embodied Cognition (New Problems of Philosophy). Routledge.

Smith, L. B. (2005). Cognition as a dynamic system: Principles from embodiment. Developmental Review, 25(3-4), 278-298.

Varela, F. J., Thompson, E., \& Rosch, E. (1992). The embodied mind: Cognitive science and human experience. MIT Press.

Wilson, A. D., \& Golonka, S. (2013). Embodied cognition is not what you think it is. Frontiers in Psychology, 4, 58.

Wilson, M. (2002). Six views of embodied cognition. Psychonomic Bulletin E Review, 9(4), 625-636.

Wilson, R. A., \& Foglia, L. (2011). "Embodied Cognition", The Stanford Encyclopedia of Philosophy. In E. N. Zalta (ed.), The Stanford Encyclopedia of Philosophy. 